\title{
A GEOMETRIC PROOF OF THE STRONG MAXIMAL THEOREM
}

\author{
BY A. CORDOBA AND R. FEFFERMAN
}

Communicated by Robert Bartle, May 25, 1975

In $R^{n}$, suppose we consider the operator $M_{s}$ given by

$$
M_{s}(f)(x)=\sup _{R} \frac{1}{|R|} \int_{R}|f(y)| d y
$$

where $f$ is a locally integrable function on $R^{n}$ and the sup is taken over all rectangles with sides parallel to the axes which contain the point $x$. Then the strong maximal theorem may be taken as the statement that $M_{s}$ is bounded from $L\left(\log ^{+} L+1\right)^{n-1}(Q)$ to weak $L^{1}(Q)$, i.e.

$$
m\left\{M_{s} f>\alpha\right\} \leqslant A_{n} \int \frac{|f(x)|}{\alpha} \log ^{n-1}\left(\frac{|f(x)|}{\alpha}+1\right) d x
$$

where $A_{n}$ is some absolute constant, and $Q$ is the unit cube in $R^{n}$.

Our result consists of a purely geometric argument establishing such an inequality. At the heart of the matter is a geometric proof of the following covering lemma:

Suppose $R_{1}, R_{2}, \ldots, R_{k}, \ldots$ is a sequence of rectangles contained inside the unit cube in $R^{n}$. Then there is a subcollection $\widetilde{R}_{1}, \widetilde{R}_{2}, \ldots$ of the $R_{k}$ 's satisfying the following conditions:

(1) $\left|\bigcup \widetilde{R}_{k}\right| \geqslant c_{n}\left|\cup R_{k}\right|$ for some absolute constant $c_{n}>0$, and

(2) $\left\|\exp \left(\Sigma \chi_{\widetilde{R}_{k}}\right)^{1 /(n-1)}\right\|_{L^{1}} \leqslant C_{n} \| \cup R_{k} \mid$ for some absolute constant $C_{n}<\infty$.

These observations lead to further results in the theory of differentiation of the integral.

\section{REFERENCES}

1. A. Cordoba, On the Vitali covering properties of a differentiation basis (to appear).

2. B. Jessen, J. Marcinkiewicz and A. Zygmund, Note on the differentiability of multiple integrals, Fund. Math. 25 (1935), 217-234.

DEPARTMENT OF MATHEMATICS, PRINCETON UNIVERSITY, PRINCETON, NEW JERSEY 08540

AMS (MOS) subject classifications (1970). Primary 42A68, 42A92; Secondary 42A18. 